\title{
Vlasov Treatment of Coherent Synchrotron Radiation from Arbitrary Planar Orbits *
}

\author{
R. Warnock ${ }^{\text {a }}$, G. Bassi ${ }^{\text {b }}$, J. A. Ellison ${ }^{\text {b }}$ \\ ${ }^{a}$ Stanford Linear Accelerator Center, Stanford University, Stanford, CA 94309 \\ ${ }^{\mathrm{b}}$ Department of Mathematics, University of New Mexico, Albuquerque, NM 87131
}

\begin{abstract}
We study the influence of coherent synchrotron radiation (CSR) on particle bunches traveling on arbitrary planar orbits between parallel conducting plates. The plates represent shielding due to the vacuum chamber. The vertical distribution of charge is an arbitrary fixed function. Our goal is to follow the time evolution of the phase space distribution by solving the Vlasov-Maxwell equations in the time domain. This provides simulations with lower numerical noise than the macroparticle method, and allows one to study such issues as emittance degradation and microbunching due to CSR in bunch compressors. The fields excited by the bunch are computed in the laboratory frame from a new formula that leads to much simpler computations than the usual retarded potentials or Lienard-Wiechert potentials. The nonlinear Vlasov equation, formulated in the interaction picture, is integrated in the beam frame by approximating the Perron-Frobenius operator. The distribution function is represented by B-splines, in a scheme preserving positivity and normalization of the distribution. For application to a chicane bunch compressor we take steps to deal with energy chirp, an initial near-perfect correlation of energy with position in the bunch.
\end{abstract}

Key words: CSR, coherent synchrotron, bunch compressor, chicane, Vlasov PACS: 41.60.-m, 41.60.Ap, 42.25.Kb, 52.65.Ff, 41.75.Fr, 41.20.-q

\footnotetext{
* Support from DOE grants DE-AC02-76SF00515 and DE-FG02-99ER1104 is gratefully acknowledged

Email addresses: warnock@slac.stanford.edu (R. Warnock), gbassi@math.unm.edu (G. Bassi), ellison@math.unm.edu (J. A. Ellison).
} 


\section{Introduction}

Coherent synchrotron radiation (CSR) is expected to play an important and often detrimental role in various advanced accelerator projects, for instance in linac-based coherent light sources [1,2] and energy recovery linacs [3]. A large concern is that CSR may cause transverse emittance growth in a bunch compressor by inducing an energy spread that is mapped into the transverse motion through dispersion. There are two principal tasks in numerical modeling of such phenomena. First, one must compute the fields produced by the particle bunch from a knowledge of past and present values of its charge/current density. Principally, the longitudinal field within the bunch is needed, to find the energy change due to the field of the bunch itself. Second, one needs to find the effect of this field in subsequent evolution of the bunch form. This second problem is usually addressed by the macro-particle method. One may aspire to various degrees of self consistency. In the simplest case one would assume that the electromagnetic field is that from particles moving under external fields alone, without self forces. One would then examine the evolution of an initial macro-particle configuration under that fixed field plus external fields. Such a calculation is not self-consistent, but it can be useful and informative. At the other extreme, one would try for full self-consistency, letting self fields act to rearrange the particles at every time step.

Codes to address these issues have been developed over the past few years [48], and despite formidable complications a decent agreement of the different codes has been found in a benchmark test proposed at a meeting in Zeuthen [9]. Nevertheless, because the problem is so complicated there is probably room for improvement in the algorithms. We report some efforts to improve the formulation in two directions: (1) to simplify the field calculation, and (2) to study multi-particle dynamics by the Vlasov equation rather than by the macro-particle method. We think that our proposal for item (1) will certainly simplify coding, and we hope that it will also speed up the computation and make it more robust. In item (2) the use of Vlasov may or may not save time, but it promises to give much less noise and orderly convergence as the mesh in phase space is refined. In fact, a Vlasov study of longitudinal motion in storage rings with CSR has been very successful $[10,11]$. That was in a twodimensional phase space but over time intervals very much longer than those of the four-dimensional, single-pass problem of a bunch compressor.

A feature of our work on both items (1) and (2) is that we apply a standard technique of numerical analysis, namely spline approximation, to represent charge/current densities and the phase space distribution function. Splines are extremely convenient and have well understood convergence properties. As far as we know, other investigators have not used general methods of numerical analysis, instead preferring special techniques such as a representation 
of the bunch as a superposition of small Gaussian macro-particles [5].

\section{Coordinate Systems and Charge/Current Densities}

We have in mind single-pass systems such as a chicane bunch compressor traversed only once by the particles [9]. With small modifications our treatment should apply as well to multi-pass systems such as storage rings. In the laboratory frame the spatial coordinates are $(Z, X, Y)$ and the independent variable is $u=c t$. The particle orbits lie between two infinite, parallel, perfectly conducting plates, which are perpendicular to the $Y$-axis and separated by a distance $h=2 g$. Points with $Y=0$ are in the midplane, and every orbit is in some plane $Y=$ const $\in(-g, g)$. The $Y$-direction is "vertical". For the chicane the reference orbit (design orbit) follows the $Z$-axis initially and finally, and is in the midplane. We write the reference orbit as $\mathbf{R}_{0}(\beta u)$ with $\mathbf{R}_{0}=\left(Z_{0}, X_{0}\right)$, where $\beta c$ is the speed of the reference particle. Generally, bold face letters refer to two- component vectors.

A point can also be specified in terms of Frenet-Serret coordinates: arc-length $s$ along the reference trajectory, and the perpendicular distance $x$ from the trajectory at $\mathbf{R}_{0}(s)$. Thus, $\mathbf{R}=(Z, X)=\mathbf{R}_{0}(s)+x \mathbf{n}(s)$ where the unit normal vector is $\mathbf{n}(s)=\left(-X_{0}^{\prime}(s), Z_{0}^{\prime}(s)\right)$ and has been chosen so that its $X$ component is positive and the corresponding unit tangent is $\mathbf{t}(s)=\mathbf{R}_{0}^{\prime}(s)=$ $\left(Z_{0}^{\prime}(s), X_{0}^{\prime}(s)\right)$. After a change of independent variable from $u=c t$ to $s$ through standard manipulations, a convenient set of dynamical variables for motion in horizontal planes consists of the "beam frame" phase space coordinates $\left(z, p_{z}, x, p_{x}\right)$. Here $z(s)=s-\beta c t(s)$, where $t(s)$ is the time of arrival at arclength $s$. Thus $z$ is the signed distance along the reference orbit, positive in front of the reference particle. The conjugate variable is the relative energy deviation $p_{z}(s)=\left(E(s)-E_{0}\right) / E_{0}$, with $E_{0}=m \gamma c^{2}$ the energy of the reference particle. Also $p_{x}(s)=v_{x}(s) / \beta c$ where $v_{x}$ is the velocity component along $\mathbf{n}$.

Clearly $Z, X$ and $u$ are determined explicitly from $z, x, s$. To obtain an explicit form for the inverse we note that we are interested in a bunch of particles with small $z$ and $x$. Expanding to lowest order in $z$ and $x$ we obtain

$$
\mathbf{R}=\mathbf{R}_{0}(z+\beta u)+x \mathbf{n}(z+\beta u)=\mathbf{R}_{0}(\beta u)+M(\beta u) \mathbf{r}+O\left(\kappa\left(z^{2}, x z\right)\right),
$$

where $\mathbf{r}=(z, x)$ and $M=(\mathbf{t}, \mathbf{n})$ is a rotation matrix. The factor $\kappa$ in the remainder is the curvature of the reference orbit and is defined by $\mathbf{n}^{\prime}(s)=$ $\kappa(s) \mathbf{t}(s)$. Since the radius of curvature is much greater than the bunch size in our cases of interest, the remainder is negligible. Thus given $Z, X$ and $u$ we have $\mathbf{r}=M^{T}(\beta u)\left(\mathbf{R}-\mathbf{R}_{0}(\beta u)\right)$ to good approximation, where $T$ denotes transpose. 
We wish to solve the Vlasov equation for the distribution function on beam frame coordinates, $f\left(z, p_{z}, x, p_{x}, s\right)$. On the other hand, it is most convenient to solve the Maxwell equations in the laboratory frame. We must then express the lab frame charge/current density in terms of $f$. To that end suppose that $f$ has unit integral over all space and define

$$
\begin{aligned}
\rho(\mathbf{r}, s) & =Q \int d p_{z} d p_{x} f\left(z, p_{z}, x, p_{x}, s\right), \\
\tau(\mathbf{r}, s) & =Q \int d p_{z} d p_{x} p_{x} f\left(z, p_{z}, x, p_{x}, s\right),
\end{aligned}
$$

where $Q$ is the total charge. To an excellent approximation the lab frame charge density $\rho_{L}$ is

$$
\rho_{L}(\mathbf{R}, Y, u)=H(Y) \rho(\mathbf{r}, \beta u), \quad \mathbf{r}=M^{T}(\beta u)\left(\mathbf{R}-\mathbf{R}_{0}(\beta u)\right)
$$

where $\int H(Y) d Y=1$ and $H(Y)$ is an arbitrary fixed vertical distribution of charge. The main approximation (beyond the use of the first order expansion $(1))$ is that at fixed $\mathbf{r}$ the density $\rho(\mathbf{r}, s)$ does not change appreciably when $s$ varies by an amount comparable to the bunch size. If in addition we assume that $f$ varies little when $s$ changes by a bunch size then we find a corresponding good approximation for the lab frame current density, which is defined by $\mathbf{J}_{L}(\mathbf{R}, Y, u)=Q H(Y) \int \mathbf{V F}(\mathbf{R}, \mathbf{V}, u) d \mathbf{V}$, where $\mathbf{V}$ is the lab frame velocity and $F$ the lab frame phase space density with unit integral. The formula is

$$
\mathbf{J}_{L}(\mathbf{R}, Y, u)=\beta c H(Y)[\rho(\mathbf{r}, \beta u) \mathbf{t}(\beta u+z)+\tau(\mathbf{r}, \beta u) \mathbf{n}(\beta u+z)],
$$

where $\mathbf{r}$ is as in (3). It is justified to expand $\mathbf{t}$ and $\mathbf{n}$ through first order in $z$. A derivation of (3) and (4) will be reported elsewhere.

\section{Field Calculation}

We calculate the electric field produced by $\left(\rho_{L}, \mathbf{J}_{L}\right)$, but averaged over the $Y$-distribution:

$$
\mathbf{E}(\mathbf{R}, u):=\langle\mathbf{E}(\mathbf{R}, \cdot, u)\rangle=\int_{-g}^{g} H(Y) \mathbf{E}(\mathbf{R}, Y, u) d Y .
$$

The averaged field can be computed much more quickly, and we believe that it will produce nearly the same dynamics in the $(Z, X)$ plane as the full field. 
To evaluate (5) we begin with the general formula for the retarded potential (scalar or vector),

$$
\begin{aligned}
& \psi(\mathbf{R}, Y, u)= \\
& \frac{1}{4 \pi} \int d \mathbf{R}^{\prime} \int d Y^{\prime} \xi\left(Y^{\prime}\right) \frac{S\left(\mathbf{R}^{\prime}, u-\left[\left(\mathbf{R}^{\prime}-\mathbf{R}\right)^{2}+\left(Y-Y^{\prime}\right)^{2}\right)\right]^{1 / 2}}{\left.\left[\left(\mathbf{R}^{\prime}-\mathbf{R}\right)^{2}+\left(Y-Y^{\prime}\right)^{2}\right)\right]^{1 / 2}},
\end{aligned}
$$

where $\xi(Y)$ is the effective vertical charge distribution needed to impose boundary conditions at the parallel plates by the method of images, namely

$$
\xi(Y)=\sum_{k=-\infty}^{\infty}(-1)^{k} H(Y-k h) .
$$

Here we assume that the support of $H(Y)$ is well within the interval $(-g, g)$. The term with $k=0$ gives the potential for free space. To average the potential over $Y$ as in (5) we replace the integration variable $Y^{\prime}$ by $\eta=Y^{\prime}-Y$ and find

$$
\begin{aligned}
& \psi(\mathbf{R}, u):=\langle\psi(\mathbf{R}, \cdot, u)\rangle= \\
& \frac{1}{4 \pi} \int d \mathbf{R}^{\prime} \int d \eta \Phi(\eta) \frac{S\left(\mathbf{R}^{\prime}, u-\left[\left(\mathbf{R}^{\prime}-\mathbf{R}\right)^{2}+\eta^{2}\right)\right]^{1 / 2}}{\left.\left[\left(\mathbf{R}^{\prime}-\mathbf{R}\right)^{2}+\eta^{2}\right)\right]^{1 / 2}}
\end{aligned}
$$

where $\Phi(\eta)=\int H(Y) \xi(Y+\eta) d Y$. For a Gaussian $H(Y)$ with rms width $\sigma_{Y}$ we suppose that $\sigma_{Y} \ll g$ and obtain

$$
\Phi(\eta)=\sum_{k=-\infty}^{\infty} \frac{(-1)^{k}}{\sqrt{2 \pi} \sigma} \exp \left(-\frac{1}{2}\left(\frac{\eta-k h}{\sigma}\right)^{2}\right), \quad \sigma=\sqrt{2} \sigma_{Y} .
$$

We assume that $\sigma$ is sufficiently small to justify replacing the Gaussians in (9) by delta functions. Thus, the averaging produces just a two-dimensional integral,

$$
\psi(\mathbf{R}, u)=\frac{1}{4 \pi} \sum_{k=-\infty}^{\infty}(-1)^{k} \int d \mathbf{R}^{\prime} \frac{S\left(\mathbf{R}^{\prime}, u-\left[\left(\mathbf{R}^{\prime}-\mathbf{R}\right)^{2}+(k h)^{2}\right)\right]^{1 / 2}}{\left.\left[\left(\mathbf{R}^{\prime}-\mathbf{R}\right)^{2}+(k h)^{2}\right)\right]^{1 / 2}} .
$$

The sources $S$ in (10) for averaged scalar and vector potentials $\phi, \mathbf{A}$ are $\rho_{L} /\left(H \epsilon_{0}\right), \mu_{0} \mathbf{J}_{L} / H$, respectively. These are expressed in terms of beam frame densities by (3) and (4). The averaged electric field is $\mathbf{E}=-\nabla \phi-\partial \mathbf{A} / \partial t$.

The integration in (10) is restricted to a very small part of the full $\mathbf{R}^{\prime}$ plane, because of the small size of the bunch, but it is awkward to locate this region owing to the fact that spatial and temporal arguments of the source both 
depend on $\mathbf{R}^{\prime}$. The bunch moves around, so to speak, during integration. The task of integration is made drastically easier if we take the temporal argument to be a new variable of integration. We first go into polar coordinates $(\zeta, \theta)$, then use the temporal argument $v$ in place of the radial coordinate $\zeta$. That is,

$$
\mathbf{R}-\mathbf{R}^{\prime}=\zeta \mathbf{e}(\theta), \quad \mathbf{e}(\theta)=(\cos \theta, \sin \theta), \quad v=u-\left[\zeta^{2}+(k h)^{2}\right]^{1 / 2} .
$$

This incidentally gets rid of the small divisor in (10), giving the potential as simply an integral over the source:

$$
\begin{aligned}
& \psi(\mathbf{R}, u)= \\
& \frac{1}{2 \pi} \sum_{k=0}^{\infty}(-1)^{k}\left(1-\delta_{k 0} / 2\right) \int_{-\infty}^{u-k h} d v \int_{-\pi}^{\pi} d \theta S\left(\mathbf{R}-\left[(u-v)^{2}-(k h)^{2}\right]^{1 / 2} \mathbf{e}(\theta), v\right) .
\end{aligned}
$$

A further bonus is that the derivatives required to construct the field from potentials act only on the spatial argument of the source (and the upper limit of the $v$-integral). We have

$$
\begin{gathered}
\mathbf{E}(\mathbf{R}, u)=-\frac{1}{2 \pi} \sum_{k=0}^{\infty}(-1)^{k}\left(1-\delta_{k 0} / 2\right)\left[2 \pi \mu_{0} c \mathbf{J}_{L}(\mathbf{R}, u-k h)+\right. \\
\left.\int_{-\infty}^{u-k h} d v \int_{-\pi}^{\pi} d \theta\left(\frac{1}{\epsilon_{0}} \nabla \rho_{L}(\hat{\mathbf{R}}, v)-\frac{\mu_{0} c(u-v)}{\left[(u-v)^{2}-(k h)^{2}\right]^{1 / 2}}(\mathbf{e}(\theta) \cdot \nabla) \mathbf{J}_{L}(\hat{\mathbf{R}}, v)\right)\right], \\
\hat{\mathbf{R}}=\mathbf{R}-\left[(u-v)^{2}-(k h)^{2}\right]^{1 / 2} \mathbf{e}(\theta) .
\end{gathered}
$$

Strictly speaking the integrand has integrable singularities for $k \neq 0$, however practically there are no points of singularity since $\nabla \mathbf{J}_{L}$ is effectively zero in a neighborhood of such points. It is very fortunate for the numerical scheme that (13) involves derivatives of the source only with respect to its spatial argument (denoted by $\nabla$ ), which will be given conveniently as derivatives of splines. A differentiable representation of the time dependence of the source, something relatively hard to achieve, is not needed. However, there is one remaining numerical difficulty in that the boundary term $2 \pi \mu_{0} c \mathbf{J}_{L}(\mathbf{R}, u-k h)$ and the integral may be nearly equal and opposite in sign for $k=0$, thus requiring a careful integration to get an accurate difference of two large terms. This striking but mysterious feature was observed in the calculation with assigned source reported in Section 5, but it may or may not happen in a self-consistent field calculation. For $k \neq 0$ there is no problem since $\mathbf{J}_{L}(\mathbf{R}, u-k h)$ is negligible for $\mathbf{R}$ near to the bunch at time $u$. 
It is quite easy to determine the effective region of the $\theta$ integration in (13). Note that the sources in (13) have significant values only for $\hat{\mathbf{R}}$ of (14) restricted to a bunch-sized neighborhood of $\mathbf{R}_{0}(\beta v)$, according to (3) and (4). For the CSR wake field at time $u$ we are interested only in $\mathbf{R}$ in a bunch-sized neighborhood of $\mathbf{R}_{0}(\beta u)$. Thus the integrand is appreciable only when

$$
\mathbf{R}_{0}(\beta u)-\mathbf{R}_{0}(\beta v)-\left[(u-v)^{2}-(k h)^{2}\right]^{1 / 2} \mathbf{e}(\theta)=O(\Delta),
$$

where $\Delta$ is a suitable measure of the bunch size. For $k=0$ and $u-v$ large compared to $\Delta$, this cannot be satisfied unless $\mathbf{e}(\theta)$ has nearly the same direction as $\mathbf{R}_{0}(\beta u)-\mathbf{R}_{0}(\beta v)$, which is to say that the domain of $\theta$ integration is tiny (and close to $\theta=0$ for a chicane with small bending angle). When $u-v$ gets close to $\Delta$ the domain expands precipitously to the full $(-\pi, \pi)$. For $k \neq 0$ the condition (15) cannot be met unless $u-v \gg k h$, so for image charges there are no contributions to the $v$-integral close to its upper limit.

\section{Integration of Vlasov Equation}

To state the Vlasov equation in the beam frame coordinates we need the single-particle equations of motion. We assume that the only external forces are from sharp-edged bending fields. To a good approximation the equations can be linearized, except for the nonlinear CSR force. Using also the fact that $\gamma$ is large (order of $10^{4}$ ) we obtain

$$
\frac{d z}{d s}=-\frac{x}{R(s)}, \quad \frac{d p_{z}}{d s}=\frac{e \mathcal{E}(\mathbf{r}, s)}{E_{0}}, \quad \frac{d x}{d s}=p_{x}, \quad \frac{d p_{x}}{d s}=\frac{p_{z}}{R(s)} .
$$

These are the equations of standard linear optics perturbed by the collective force from CSR, as in [12]. Here $R$ is the bending radius and its sign must be taken consistent with the definition of $\kappa$ and the sign of the external magnetic field. Also $\mathcal{E}(\mathbf{r}, s)=\mathbf{t}(s) \cdot \mathbf{E}\left(\mathbf{R}_{0}(s)+x \mathbf{n}(s), s / \beta\right)$ is the tangential component of the electrical field. We defined the field as a function of $u=c t$ rather than as a function of $s$, so we properly should use $\mathbf{E}\left(\mathbf{R}_{0}(s)+x \mathbf{n}(s),(s-z) / \beta\right)$. Since in integration of the Vlasov equation we freeze the field over times much longer than typical $z / c$, this refinement would be useless as well as expensive. We have neglected the transverse electric and transverse magnetic force, since for relativistic particles one is expected to cancel the other to high accuracy. The validity of this neglect will be checked at a later stage.

The unperturbed version of (16) with $\mathcal{E}=0$ can be solved explicitly [12] in terms of the lattice functions $D(s), D^{\prime}(s), R_{56}(s)$. This gives the transport map $\Phi(s \mid 0)$ from $s=0$ to arbitrary $s$, with inverse $\Phi(0 \mid s)$. For the Vlasov 
formulation we use the initial conditions of the unperturbed motion as phase space variables [12]. With $\zeta=\left(z, p_{z}, x, p_{x}\right)$, the phase space coordinates will be

$$
\zeta_{0}=\Phi(0 \mid s) \zeta
$$

Now $\zeta_{0}$ is constant in the unperturbed case, and possibly slowly varying in presence of the perturbation. The Vlasov equation for the distribution function $g\left(\zeta_{0}, s\right)=f(\zeta, s)$ is

$$
\frac{\partial g\left(\zeta_{0}, s\right)}{\partial s}+\frac{\partial g\left(\zeta_{0}, s\right)}{\partial p_{z}} \frac{e}{E_{0}} \mathcal{E}\left(\left[\Phi(s \mid 0) \zeta_{0}\right]_{1},\left[\Phi(s \mid 0) \zeta_{0}\right]_{3}, s ; g\right)=0
$$

The Vlasov equation is to be integrated by approximating the Perron-Frobenius $(\mathrm{PF})$ operator. Over a small interval $(s, s+\Delta s)$ we regard $\mathcal{E}(\mathbf{r}, s ; g)$ as independent of $s$, having the value it had after the previous $s$-step, and solve the initial value problem for the single-particle equations corresponding to (18) on that interval. We represent the solution of the IVP as a map $\Psi(s+\Delta s \mid s)\left(\zeta_{0}\right)$ that takes any initial $\zeta_{0}$ into its image under the flow. The inverse map is $\Psi(s \mid s+\Delta s)$. The propagation of $g$ is given by the $\mathrm{PF}$ operator $\mathcal{P}$ associated with $\Psi$, which is to say

$$
g\left(\zeta_{0}, s+\Delta s\right)=\mathcal{P} g\left(\zeta_{0}, s\right)=g\left(\Psi(s \mid s+\Delta)\left(\zeta_{0}\right), s\right)
$$

To apply this method we need some finite-dimensional representation of $g$ and a numerical integration of the differential equations to produce $\Psi(s \mid s+\Delta s)$. For the former we choose an expansion in a spline basis made of Kronecker products of one-dimensional B-splines [13]. Rather than using splines that interpolate values of the function at mesh points, which are expensive to compute and do not guarantee positivity of $g$, we use Schönberg's Variation Diminishing Approximation, in which the coefficients are evaluated more quickly as values of the function at averages of knots. In one dimension this is

$$
f(x)=\sum_{i} f\left(x_{i}^{*}\right) B_{i}(x), \quad x_{i}^{*}=\frac{1}{k-1}\left(x_{i+1}+\cdots+x_{i+k-1}\right),
$$

for $B$ splines $B_{i}$ of order $k$ and knot sequence $\left\{x_{j}\right\}$. The approximation has second order accuracy for any $k$, so that the choice of $k$ just controls the smoothness. Since B-splines are non-negative, this method ensures that positive data $f\left(x_{i}^{*}\right)$ produce a positive function. Our algorithm will be to start with a positive distribution and redetermine the Schönberg spline coefficients after each application of the PF operator. This guarantees that the distribution stays positive. It is also important to guarantee conservation of probability, $\int g d \zeta_{0}=1$, which we do by dividing each new spline by its integral. 
For integration of the single-particle equations to find $\Psi(s \mid s+\Delta s)$ we apply Euler's method for a first try in code development, but there are various possibilities for improvement. A factorization into maps moving the point along coordinate axes in phase space may be efficient for evaluating the $\mathrm{PF}$ operator on the Kronecker product of B-splines.

Now consider the particular case of a bunch compressor, similar to that of the LCLS design. The initial distribution has the form

$$
f\left(\zeta_{0}, 0\right)=\frac{1}{2 \pi \epsilon_{0}} \exp \left[-\frac{1}{2 \epsilon_{0} \beta_{0}}\left(x_{0}^{2}+\left(\alpha_{0} x_{0}+\beta_{0} p_{x 0}\right)^{2}\right)\right] \mu\left(z_{0}\right) \rho_{c}\left(p_{z 0}, z_{0}\right)
$$

where $\beta_{0}, \alpha_{0}, \epsilon_{0}$ are initial Twiss parameters and $x$-emittance. The factor $\rho_{c}$ expresses "energy chirp", a close correlation of energy with longitudinal position in the bunch:

$$
\rho_{c}\left(p_{z 0}, z_{0}\right)=a \exp \left[-\frac{1}{2 \sigma_{u}^{2}}\left(p_{z 0}-u z_{0}\left(1+C\left(z_{0}\right)\right)\right)^{2}\right] .
$$

Here $u$ is the slope of the correlation line at $z=0, \sigma_{u}$ is a small parameter expressing lack of the desired perfect correlation, $a$ is for normalization, and $z_{0} C\left(z_{0}\right)$ is a small nonlinearity in the correlation, significant at the ends of the $z_{0}$-distribution and well represented as cubic. This nonlinearity represents effects of wake fields in the linac. The function $\mu\left(z_{0}\right)$ is approximated as parabolic over an interval, and zero elsewhere. Because of the tight correlation, the $p_{z 0}$ distribution will also be nearly parabolic.

The $p_{z 0}-z_{0}$ correlation presents a previously unmentioned problem in the spline representation of the distribution. The function $\rho_{c}$ is a prime example of functions that cannot be represented efficiently through a Kronecker product basis $\left\{B_{i}\left(z_{0}\right) B_{j}\left(p_{z 0}\right)\right\}$. To avoid the problem at $s=0$ all we have to do is use $p_{z 0}-u z_{0}$ as one phase space variable, and some independent linear combination of $p_{z 0}$ and $z_{0}$ as the complimentary variable, then use a Kronecker product basis in the new variables. Since we work in the interaction picture, we expect that the distribution of the two new variables will not change drastically with $s$, and therefore the spline representation will be efficient at all $s$. A convenient coordinate change $\left(z_{0}, p_{z 0}\right) \rightarrow\left(w_{1}, w_{2}\right)$ is the one that diagonalizes the quadratic form in the exponent of $\rho_{c}\left(\right.$ with $\left.C\left(z_{0}\right)=0\right)$ :

$$
\frac{1}{\sigma_{u}^{2}}\left(p_{z 0}-u z_{0}\right)^{2}=\left(1+u^{2}\right) w_{1}^{2},\left[\begin{array}{c}
z_{0} \\
p_{z 0}
\end{array}\right]=b\left[\begin{array}{cc}
-u & 1 \\
1 & u
\end{array}\right]\left[\begin{array}{l}
w_{1} \\
w_{2}
\end{array}\right], b=\frac{\sigma_{u}}{\left(1+u^{2}\right)^{1 / 2}}
$$

Now the $w_{1}-w_{2}$ dependence of the initial distribution is $\exp \left(-\left(1+u^{2}\right) w_{1}^{2} / 2\right)$ 
$\cdot \mu\left(b\left(w_{2}-u w_{1}\right)\right)$ which can easily be represented by Kronecker products. Then our distribution will be

$g\left(w_{1}, w_{2}, x_{0}, p_{x 0}, s\right)=\sum_{i j k l} g\left(w_{1 i}^{*}, w_{2 j}^{*}, x_{0 k}^{*}, p_{x 0 l}^{*}, s\right) B_{i}\left(w_{1}\right) B_{j}\left(w_{2}\right) B_{k}\left(x_{0}\right) B_{l}\left(p_{x 0}\right)$,

with a reasonable choice of spline knots. We allow B-splines of different phase space variables to have different knot sequences, but do not show that in the notation. It may be useful, if not strictly necessary, to diagonalize and scale the initial distribution in $\left(x_{0}, p_{x 0}\right)$ as well.

The densities (2) will also be represented as Schönberg B-spline series, but in the original coordinates with basis functions $B_{i}(z) B_{j}(x)$. The coefficients for every $s$-step must be stored, at least until the early densities can no longer communicate by light rays with the relevant evaluation points $(\mathbf{R}, u)$ of the field.

\section{$5 \quad$ Preliminary Numerical Studies}

We have concentrated to date on the crucial matter of field computation, taking an assigned charge/current source rather than one determined selfconsistently. The source in (13) is determined from (21) evolved in time by the backward linear map $\Phi(0 \mid s)$. All numerical calculations reported here take $\mu\left(z_{0}\right)$ to be Gaussian, $C\left(z_{0}\right)=0$ and use benchmark bunch compressor parameters from [9], i.e. $\gamma=9785, \epsilon_{0} / \gamma=1 \mathrm{~mm}-\mathrm{mrad}, \beta_{0}=40 \mathrm{~m}, \alpha_{0}=2.6$, $\sigma_{z 0}=200 \mu \mathrm{m}, \sigma_{u}=2 \times 10^{-6}, u=-36 \mathrm{~m}^{-1}, Q=1 \mathrm{nC}$. The evolution of the charge density in the $Z-X$ plane in the absence of CSR is shown in Fig.(1). We have focused on calculations of the mean and variance of the relative energy loss, the variance of the relative energy deviation and the normalized transverse emittance (x-emittance) for comparison with [9]. We report results on the first two. We calculate these in a first order perturbation theory where we integrate the equations of motion (16) with initial conditions at $s=0$ chosen randomly according to (21), (22) and using the field $\mathcal{E}$ from the assigned charge/current source.

The relative energy loss, $p_{z L}(s)$, for a single particle is given by

$$
p_{z L}(s)=p_{z}(s)-p_{z 0}=\frac{e}{E_{0}} \int_{0}^{s} \mathcal{E}\left(\mathbf{r}\left(s^{\prime}\right), s^{\prime}\right) d s^{\prime}
$$

where $\mathbf{r}(s)$ is determined from (16), and depends on the initial conditions, and $p_{z 0}$ is the unperturbed relative energy deviation which is constant. Since 
the standard deviation of $p_{z L}$ is roughly $2 \times 10^{-4}$, the statistical error with $n$ particles is $2 \times 10^{-4} / \sqrt{n}$. Thus we have taken 400 particles giving a statistical error of order $10^{-5}$.

Our result for no shielding is shown by the solid (blue) curve in Fig.(2) where we plot $\left\langle p_{z L}\right\rangle$ vs $s$. Note our statistical error of $10^{-5}$ is very small on the scale of the figure. The self-consistent, no shielding calculation of Kabel [6], [9] is also shown on the figure by the dotted (red) curve and the agreement is good. Nevertheless we expect that our self-consistent calculation will make changes in the result. By the dashed (green) curve we plot our result including shielding, for interplate gap $h=1 \mathrm{~cm}$. We added only the contribution from $k=1$ which we believe to be dominant.

In Fig. (3) we show our results for the standard deviation $\operatorname{SD}\left(p_{z L}(s)\right)=$ $\left\langle\left(p_{z L}(s)-\left\langle p_{z L}(s)\right\rangle\right)^{2}\right\rangle^{1 / 2}$ of $p_{z L}$ by the dashed (blue) curve. The solid (red) curve is the negative of $\left\langle p_{z L}(s)\right\rangle$ with no shielding from Fig. (2). As in Fig. (2) $\mathrm{SD}\left(p_{z L}\right)$ was computed using 400 particles only as fluctuations for this number of particles were small. In Fig. (4), the results of Emma, [6], [9], are plotted similarly. The agreement is reasonable given that Emma's field calculation involves simplifications that we have not invoked; for instance his charge/current source is one-dimensional.

We do not show our preliminary results for increase in the normalized transverse emittance due to CSR, but we have evidence that they are consistent with the results shown in [6], [9].

We continue to work on refining the above results, in particular the emittance. In addition it will be interesting to repeat our calculations with realistic $C\left(z_{0}\right)$ and $\mu\left(z_{0}\right)$. The effect of $C$ has big practical importance. The assigned source model is a good guide of what to expect in the field calculation with selfconsistency. In fact, we plan to use it to determine the regime in which the $\theta$-integrand of (13) is appreciable, using analytic formulas to make precise the considerations at the end of Section 3. In Fig.(5), for $u=12.75$ at the center of the fourth magnet, we show that the important range of $\theta$ is very small for most $v$, but spreads out precipitously to the full $(-\pi, \pi)$ when $v$ gets close to $\mathrm{u}$. This means that the $v$-integrand also shoots up, roughly as a hyperbola. It is then useful to change integration variable $v \rightarrow \xi=-\log (u-v+\epsilon)$, with a small positive $\epsilon$, to spread out the peak. A typical $\xi$-integrand is shown in Fig.(6). Except for the small blip near $\xi=-1.6$, which is easy to locate because it is near the retarded time, this can easily be integrated on a uniform mesh. 


\section{Acknowledgments}

Conversations with P. Emma, A. Kabel, and M. Venturini are gratefully acknowledged.

\section{References}

[1] LCLS Design Study Report, SLAC, 1998.

[2] TESLA X-ray FEL project. See http://tesla-new.desy.de.

[3] For an overview see J. B. Murphy, Proc. 2003 Part. Accel. Conf., p.176, paper TOAC001.

[4] R. Li, Nuc. Instrum. Methods Phys. Res., Sect.A 429, 310 (1998).

[5] A. Kabel, M. Dohlus, and T. Limberg, Nuc. Instrum. Methods Phys. Res., Sect.A 455,185 (2000); M. Dohlus, A. Kabel, and T. Limberg, ibid. 445, 338 (2000).

[6] L. Giannessi and M. Quattromini, Phys. Rev. ST Accel. Beams 6, 120101 (2003).

[7] M. Borland, Argonne National Laboratory report APS LS-287 (2000).

[8] P. Emma, private communication, and G. Stupakov and P. Emma, Proc. 2002 Euro. Part. Accel. Conf., p.1479.

[9] ICFA Beam Dynamics Mini-Workshop on CSR, Berlin-Zeuthen, 2002. See http//www.desy.de/csr.

[10] M. Venturini and R. Warnock, Phys. Rev. Lett. 89, 224802 (2002).

[11] M. Venturini, R. Warnock, R. Ruth, and J. A. Ellison, "Coherent Synchrotron Radiation and Bunch Stability in a Compact Storage Ring", submitted to Phys. Rev. ST Accel. Beams.

[12] S. Heifets, G. Stupakov, and S. Krinsky, Phys. Rev. ST Accel. Beams, 5, 064401 (2002).

[13] C. de Boor, A Practical Guide to Splines, (Springer, New York, 2002). 


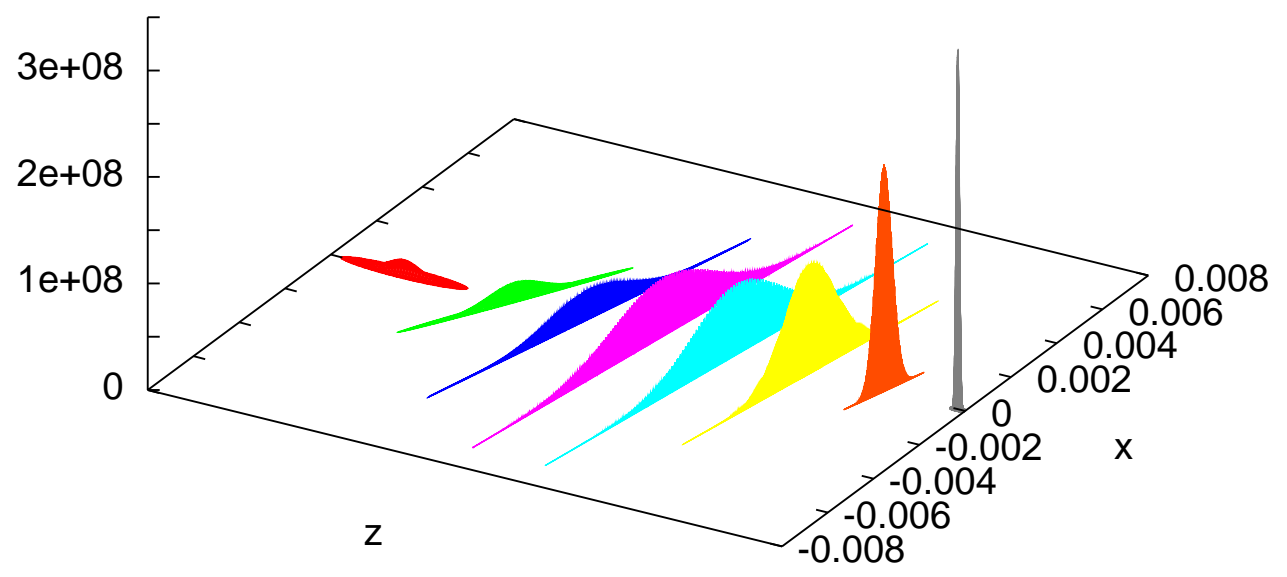

Fig. 1. Charge density in $Z-X$-plane at successive times, showing bunch compression.

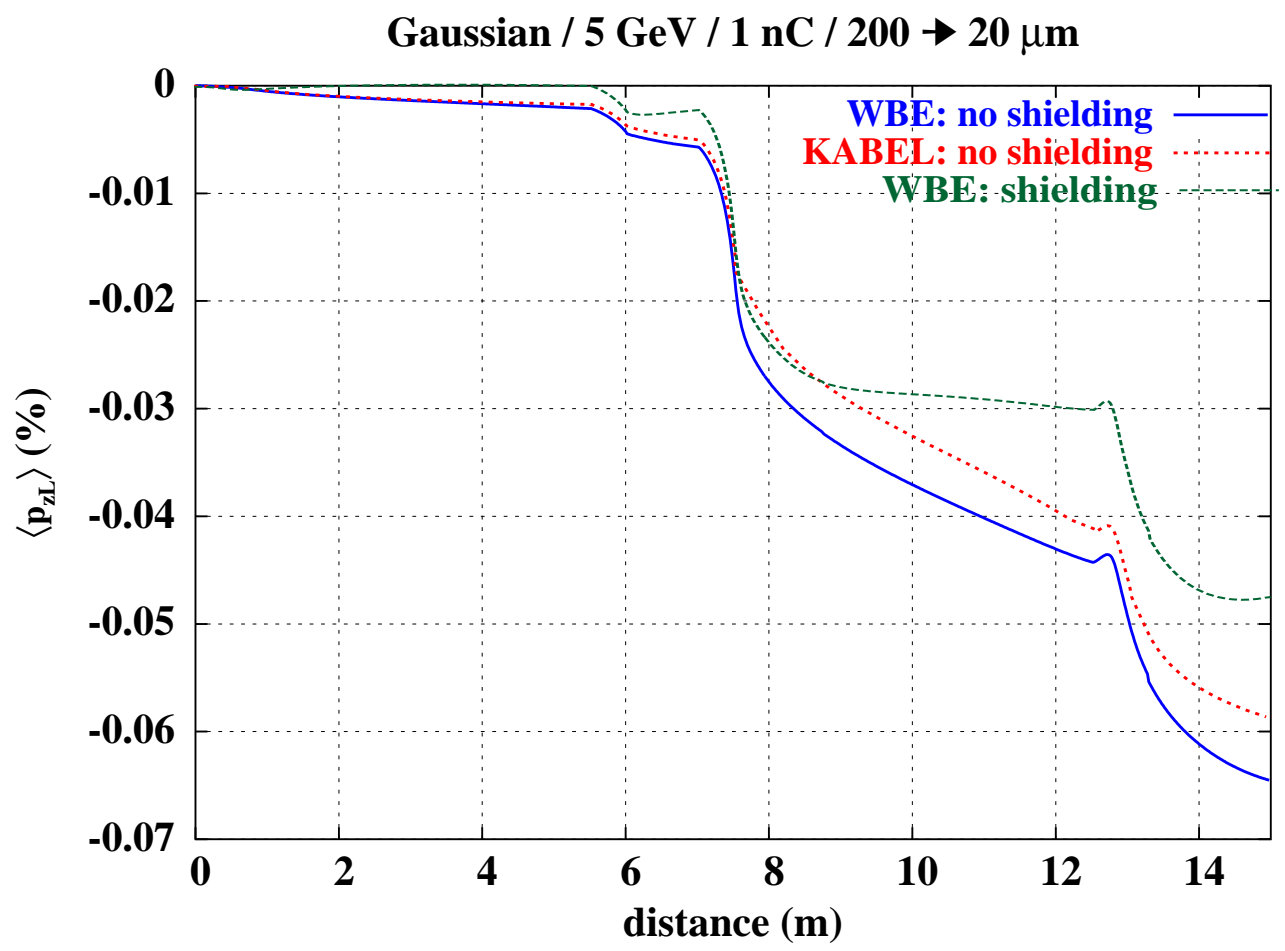

Fig. 2. Mean relative energy loss, $\left\langle p_{z L}\right\rangle$, vs. position in chicane. 


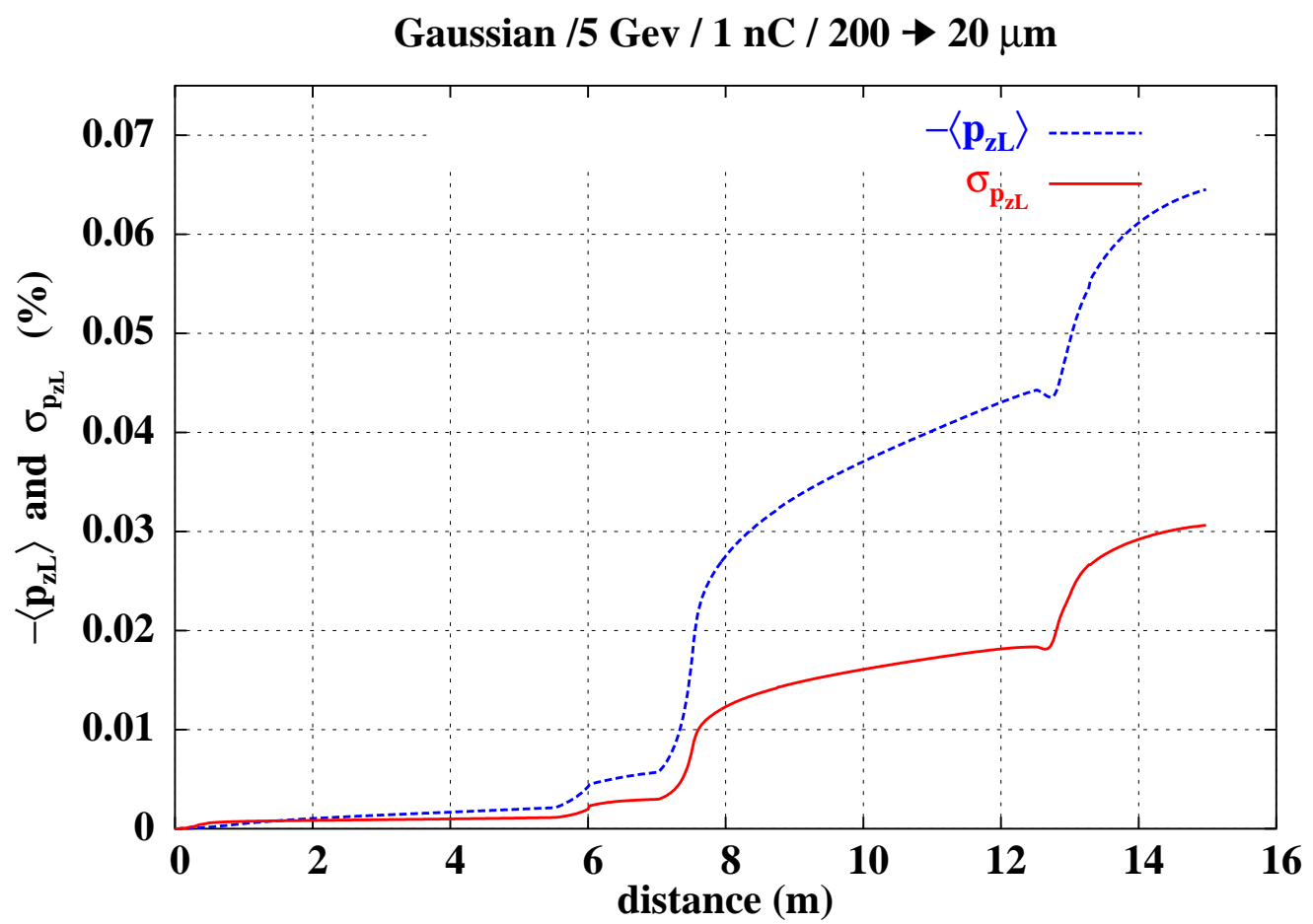

Fig. 3. Negative of $\left\langle p_{z L}\right\rangle$ (dashed blue line) and $\operatorname{SD}\left(p_{z L}\right)$ (solid red line) vs. position in chicane.

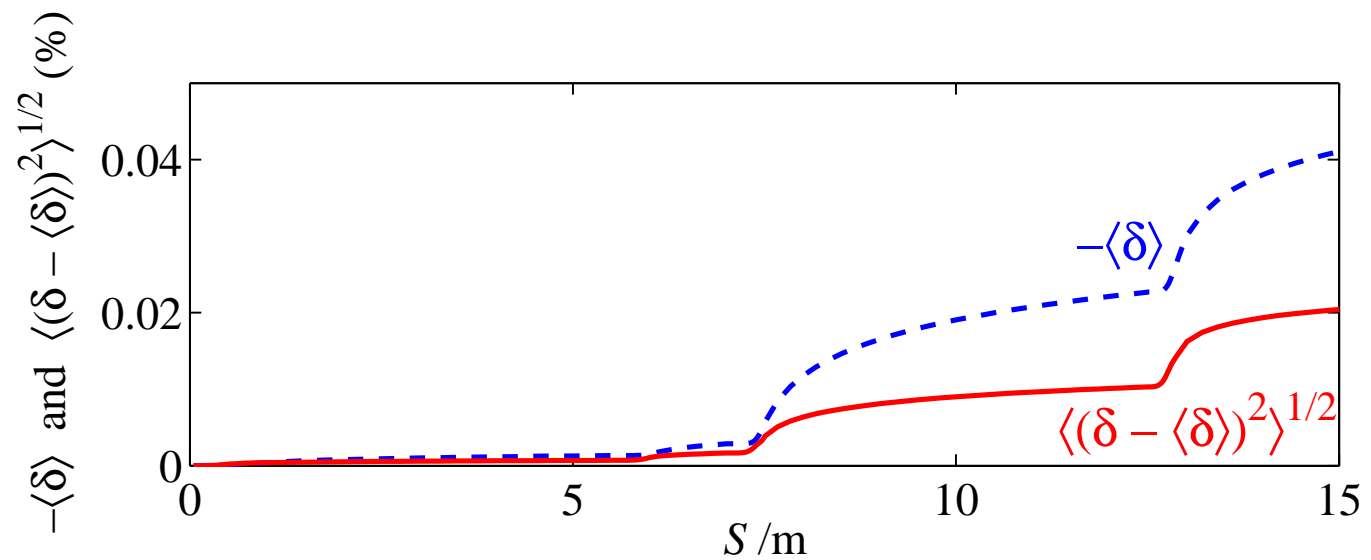

Fig. 4. Same as Fig. (3) for results of Emma [6], [9]. Here $\delta=p_{z L}$.

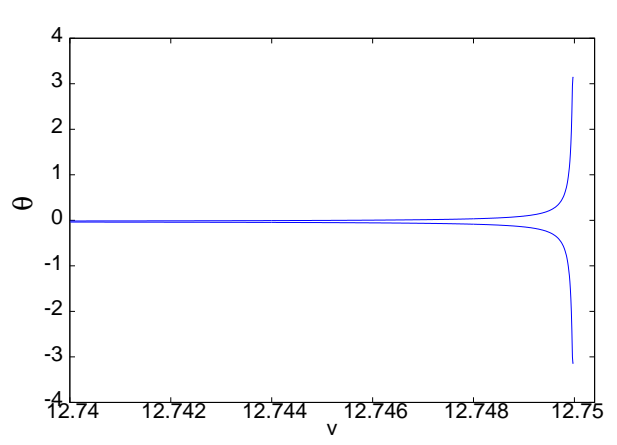

Fig. 5. Domain of $\theta$ integration, between the two curves.

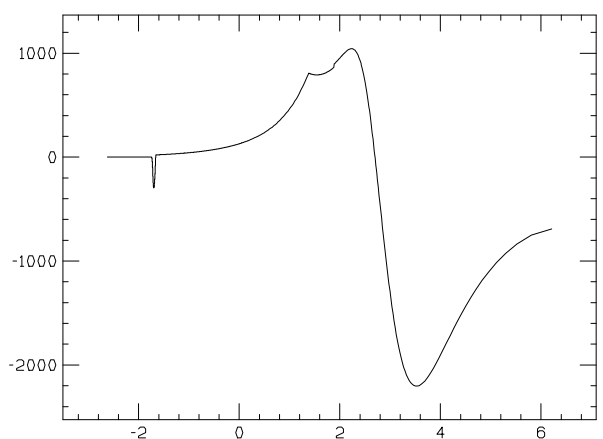

Fig. 6. $\xi$-integrand. 